\title{
La educación ante una pandemia: claves iniciales para superar brechas y poner manos a la obra
}

Elena Duce Pastor

\begin{abstract}
Resumen
En este artículo se propone una revisión del perfil del alumno y del profesor, a raíz del paso de la formación presencial a en línea, de manera masiva. Dado que la pandemia de la coviD-19 ha transformado en días la manera de aproximarnos a la educación, se ofrecen una serie de pautas para organizar las clases y conseguir efectividad en la respuesta del alumnado. Para ello, se usa la metodología de análisis de los perfiles del mundo digital y se abordan las posibles brechas que tiene que superar el docente.
\end{abstract}

Palabras clave: educación, brecha digital, emergencia, nativos digitales, profesores, coviD-19.

\section{EDUCATION IN THE FACE OF A PANDEMIC: INITIAL KEYS TO BRIDGE GAPS AND GET TO WORK}

\begin{abstract}
This article presents a revision of students and teachers' profiles, due to the change from face-to-face education to online classes, in a massive way. Since covid-19 pandemic has transformed education in a few days, some guidelines are offered, with the propose of organizing classes and exercises in order to achieve effectiveness in students' response. To pursue the task, it is necessary to consider several profiles in our digital world and the possible difficulties that teachers must be overcome.
\end{abstract}

Keywords: education, digital divide, emergency, digital natives, teachers, covid-19. 
Licenciada en Historia, graduada en Ciencias y Lenguas de la Antigüedad por la Universidad Autónoma de Madrid. En 2019 consigue su doctorado, con la máxima calificación, con una trabajo sobre el matrimonio en la Grecia Antigua.

También cuenta con un posgrado en innovación docente universitaria y con publicaciones sobre innovación docente. Actualmente funge como profesora de educación secundaria. Sus ámbitos de interés son los estudios de género, las relaciones familiares en la Antigüedad y el legado clásico de la Antigüedad y sus usos en el alumnado, especialmente desde el punto de vista del cine y del cómic.

\section{Introducción}

Desde enero de 2020 diversos países de todo el mundo se han visto obligados a paralizar sus actividades debido a la pandemia provocada por el SARS-CoV-2, un virus para el cual aún no existe una vacuna. La necesidad de mantener la distancia social y aislar a las familias en sus casas, durante un perío do de tiempo indeterminado, ha cambiado el modelo educativo de presencial a la modalidad en línea. La educación, que cuenta con una gran tradición presencial y grupal, ha sido uno de los sectores que han tenido que ponerse manos a la obra para encontrar una solución.

Como profesora española, después de haber tenido que concluir un curso mediante docencia en línea, y enfrentándonos a la misma situación en el presente ciclo, es interesante hacer una reflexión sobre las dificultades de los perfiles del alumno y del profesor ante la pandemia, y acerca de estrategias que puedan ser aplicadas ante la emergencia. Los objetivos de este artículo son la identificación de los principales problemas de los profesores y los alumnos, así como de las brechas digitales posibles, para generar un contexto adecuado de aprendizaje.

Como profesora de educación secundaria, me he enfrentado a los problemas del alumnado en estas circunstancias, tanto académicos como personales. Por ello, ofrezco una serie de propuestas para promover un aprendizaje significativo en los alumnos, basadas en su propio perfil como nativos digitales. La novedad consiste en tener en cuenta las excepcionales circunstancias en las que trabajamos en este momento y que influyen en su aprendizaje. Principalmente, por un lado, hemos debido acortar los tiempos de formación para adaptarnos al cambio, y, por otro, las circunstancias psicológicas del alumnado se han visto afectadas por procesos traumáticos como el confinamiento o el contacto estrecho con la muerte. 


\section{El modelo de alumno y profesor del momento actual}

Para poder iniciarnos en el análisis del problema, debemos tener en cuenta el perfil del alumnado y del profesorado que tenemos en la actualidad, y que está relacionado con las nuevas tecnologías, y el problema y las diferencias de acceso a las mismas, que se denominó brecha digital.

La brecha digital fue una cuestión que ocupó a los Estados en los años noventa del pasado siglo. Hoy en día, ha sido prácticamente superada gracias al uso de internet en los dispositivos móviles. No obstante, ha sido sustituida por otras brechas, como la del aprovechamiento de la información contenida en la red (Duart, 2012), que genera distracciones constantes en los usuarios. Es precisamente la distracción del alumnado delante de una pantalla lo que debemos combatir, a través de un estímulo controlado y enfocado al aprendizaje.

La disponibilidad de información en la red es inmensa, pero el trabajo de selección y, sobre todo, el aprendizaje previo para llegar a ella supone un proceso largo, tiempo del que no se dispone en una situación de emergencia. El alumnado no ha adquirido las capacidades necesarias para acceder a la información adecuada y el profesor lo puede realizar en plazos muy limitados de tiempo. Por lo tanto, se hace indispensable el aprovechamiento de recursos de conocimiento ya consolidados, para poder sostener un aprendizaje significativo.

Debemos dejar de lado la visión romántica de la educación a distancia como un recurso accesible para todos, así como los mitos asociados a la misma, como la supuesta igualdad y objetividad (Cabero, 2014). La formación a distancia es un concepto amplio que abarca desde los cursos de formación permanente — donde el alumno gestiona su tiempo-, a lo que nos enfrentamos en este momento: una situación derivada de una pandemia que nos obliga a cambiar la forma de enseñar. Se presentan evidentes problemas cuando se pasa de un día a otro a la modalidad en línea sin una gestión previa. El docente debe preparar materiales y recursos para conseguir un aprendizaje significativo, especialmente en el ámbito en el que se mueve esta propuesta, que es el de la educación obligatoria en niños y adolescentes. Por ello, me voy a centrar en el proceso de transición, rápido y repentino, para poder establecer propuestas de mejora, centradas en el alumnado de educación obligatoria.

\section{La rápida adaptación}

Lo primero que debemos mencionar es la necesidad de un cambio rápido. El paso de la docencia presencial a la docencia en línea no es una acción voluntaria o una experiencia piloto, sino una decisión tomada de la noche a la mañana por la clase política. El docente y el alumnado simplemente se adaptan a las circunstancias, pues no han decidido libremente pasarse a esta modalidad. Por lo tanto, no hay tiempo de formación; de ser necesaria ésta se ha de hacer sobre la marcha con los recursos que el propio profesor pueda conseguir. 
En resumidas cuentas, tanto el docente como el alumno necesitan contar con una experiencia anterior que les ayude al menos a empezar: estos conocimientos derivan de la relación previa con las tecnologías. En este momento debemos distinguir dos tipos de perfiles, separados por su edad y por su nacimiento en relación con las tecnologías digitales.

La terminología de nativo y emigrante digital es una trasposición de los estudios de Antropología de Margaret Mead en 1935, de clasificación de las sociedades desde un punto de vista de su inclusión en el mundo moderno. En 2001, Prensky lo adapta a las nuevas tecnologías, acuñando el término de nativo digital para designar a la persona que desde su más tierna infancia había estado en contacto con las tecnologías y, por lo tanto, estaba adaptado a ellas. Si bien parece sorprendente utiliazar terminología creada en el siglo xIx, esto tiene cierto sentido pues la tecnología ha sido insertada en la sociedad como un aspecto foráneo, de manera rápida y expansiva. Los nativos digitales han aprendido algo que les era ajeno, siendo más imitadores de conductas que creadores de ellas. La brecha es de tipo cognitivo, ya que el aprendizaje a edad temprana es mucho más rápido y significativo (Piscitelli, 2009). Por contraposición, el emigrante digital es el que tiene que moverse hacia al cambio, en este caso, el prototipo del docente. Con el uso de esta terminología definiremos el perfil del alumno y del profesor.

Los alumnos son los denominados nativos digitales. Han "nacido" con una pantalla táctil en la mano y tienen la intuición de haber crecido al tiempo que se producían las innovaciones. Esto los hace ser tremendamente visuales, efectivos en el manejo de las aplicaciones, pero también poco constantes. Una pantalla es un elemento de ocio y ahora deben acostumbrarse a usarlo como objeto de trabajo. Los nacidos después del año 2000 son los primeros que han aprendido no sólo contenidos, sino elementos básicos del propio crecimiento intelectual, como la subjetividad a través de internet (Piscitelli, 2006). Si bien, al inicio, la sociedad se ha mostrado escéptica y crítica sobre el tiempo que pasaban los alumnos delante de una pantalla, ahora éstos tienen una ventaja indiscutible con la que no cuentan los docentes que han de ser efectivos en estos momentos. Sin embargo, esta situación no implica necesariamente que los alumnos sepan aprender a través de la tecnología, ya que hasta ahora han hecho un uso lúdico de ella.

Los docentes, cuya edad oscilará entre los 25 y los 65 años, no han nacido en un mundo digital, si bien los jóvenes gozan de una ventaja considerable. La adaptación al uso de internet ha sido progresiva en todas las edades, aunque 
hay diferentes resultados. Para los docentes a punto de jubilarse, probablemente su experiencia digital quede reducida a un smartphone y quizá un ordenador portátil en casa, mientras que los más jóvenes suelen tener diversos aparatos electrónicos y están familiarizados con las nuevas tecnologías. De esta manera, se puede decir que hay un problema de inseguridad en los docentes, ya que, por norma general, los alumnos están más aventajados que el propio profesor, que tarda más tiempo en aprender programas, que en cuanto llegan a dominar son sustituidos por uno nuevo. Esto genera una frustración inicial del maestro, que además podría sentir resistencia al cambio y despreciar las nuevas tecnologías, considerándolas una forma de distracción para el alumnado (Cabero, 2014).

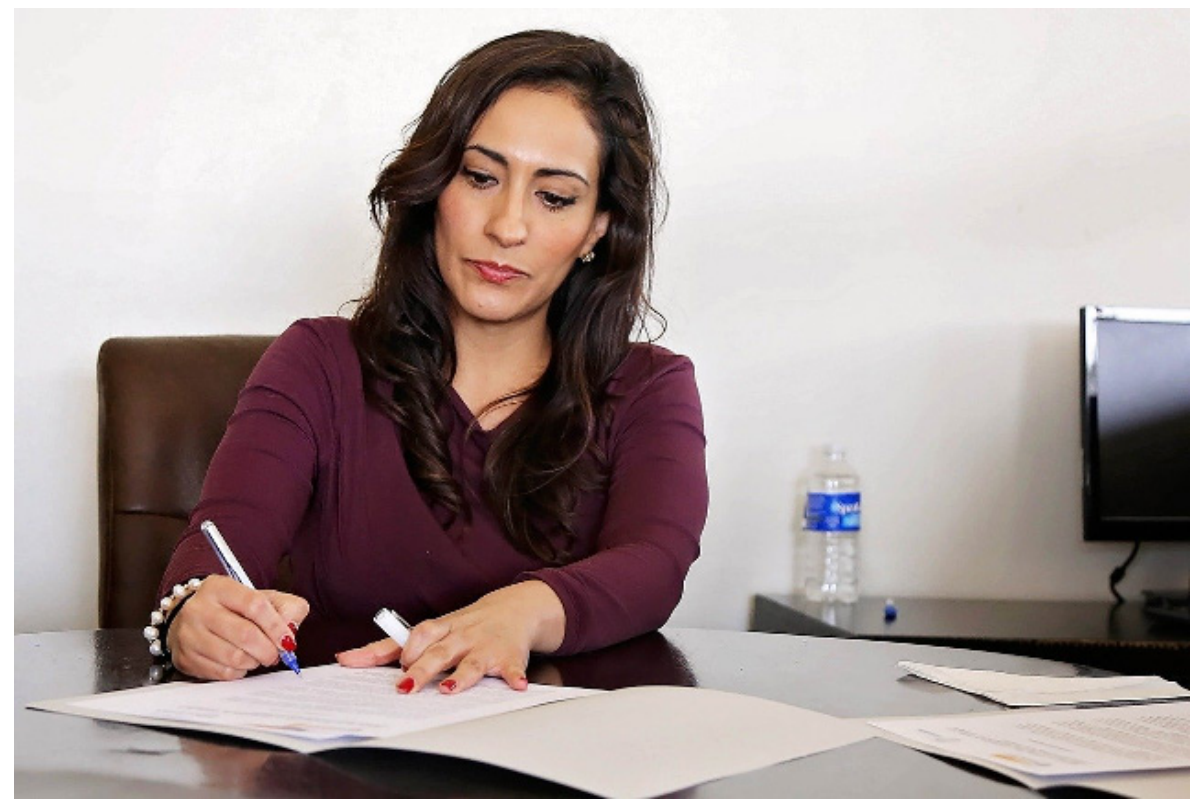

A lo anterior se le debe añadir que la disponibilidad de un ordenador no está garantizada. Debemos distinguir el acceso a internet y las tic en dos modalidades. Por un lado, modalidad smartphone, ya que hoy en día es el aparato electrónico más democrático y, por otro, en el ordenador, pues no todos los hogares tienen acceso a un dispositivo o a una conexión apropiada (Lamschtein, 2010).

Los alumnos probablemente tendrán más disponibilidad de usar su smartphone de manera constante, mientras que el ordenador puede estar siendo usado por sus progenitores que tienen que teletrabajar.

\section{Medidas a considerar para una puesta en marcha efectiva}

Si algo hay que tener en cuenta es la importancia de conocer las habilidades que los alumnos han desarrollado previamente a este momento. Como nota Johnson, (2005), la sleeper curve incluye todo tipo de conocimientos que hasta ahora no han sido reconocidos, pero que han hecho más complejo el proceso mental de los seres humanos gracias a los medios de comunicación de masas. Por lo tanto, es ese el punto de vista que hay que tomar. 


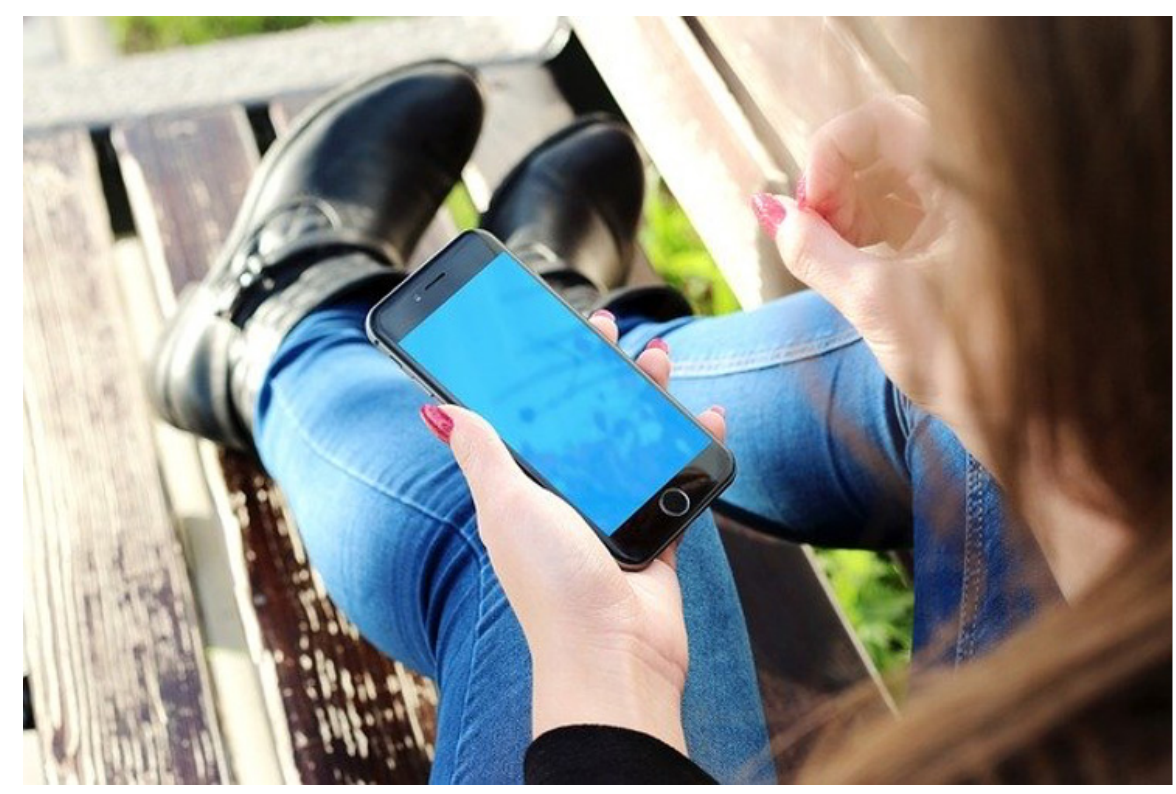

En primer lugar, hay que reflexionar sobre las tecnologías que ya manejan y el modo de acceso a la información. Las actividades deben contemplar la visualidad, brevedad y dinamismo de las redes sociales. De cara al nuevo curso, el uso de cuestionarios de diagnóstico permite a los docentes tener información relevante sobre el manejo de aplicaciones, para poder desarrollar actividades planificadas. Minimizar el ensayo-error en estos momentos nos dará seguridad y romperá esa resistencia al cambio.

En segundo lugar, es esencial reconvertir la relación del alumno con los medios digitales al ámbito educativo. Para ello, es necesario comprender que el alumnado ya ha acudido a la tecnología como un medio informal de formación (Crovi, 2010). A través de las redes sociales o canales como YouTube, los alumnos descubren la forma de solucionar problemas diarios, desde la instalación de un programa o coser el bajo de un pantalón. Lo importante es tomar ese bagaje previo y ordenarlo en una secuencia de contenidos seleccionados por el profesor, pero siempre usando el mismo lenguaje.

Dividir las tareas, añadir pausas y transformar los ritmos serán grandes aliados. Es mejor usar dos vídeos cortos que uno largo en las tareas, así como fomentar no sólo el reciclaje de recursos en red, sino elaborar materiales propios con la duración adecuada.

Para favorecer la higiene en el trabajo de los alumnos, es recomendable el uso de una única plataforma digital para el centro educativo, donde cada profesor tenga un espacio para ordenar sus contenidos. Toda la información ha de estar en el mismo servidor para que los alumnos sean capaces de dividir el espacio digital educativo del espacio de ocio. Es importante tener en cuenta la privacidad de los alumnos y los derechos de autor, para no exponer a los primeros a posibles ataques y respetar las leyes de protección de datos. 


\section{Fomentar la concentración}

Estar cerca del smartphone les hace estar visibles en sus redes sociales, recursos que normalmente son un distractor. Por lo tanto, hay que plantear la generación de contenidos que les alejen de ellas. Los medios tecnológicos usados por los alumnos para aprender ya para distraerse se concentran entre sí, es decir, a solo una pestaña de su ordenador o un click de su smartphone estará el aula virtual y su cuenta personal en redes sociales (Busquet y Uribe, 2011). Los distractores están al lado de las herramientas. Por ello, lo mejor es proponer tareas no muy largas, que usen la misma tecnología a la que están acostumbrados, fomentando la inmediatez y la tarea corta. Al mismo tiempo, convendrá llevar un control del uso de internet con la colaboración de los padres; de nuevo es importante dividir tiempos y espacios digitales, separar el ocio del aprendizaje.

\section{Reclamar las entregas en tiempos cortos}

La recompensa rápida y efímera forma parte de las redes sociales, por lo tanto, la comunicación constante hará más efectivo el seguimiento, especialmente si es a largo plazo. Para este punto hace falta compromiso por parte del profesor. Aunque pueda ser engorroso, hay que fijar tiempos cortos de respuesta y cumplirlos, para que las tareas del alumnado no se pierdan en semanas de espera de correcciones. La desorganización de las tareas y la carga excesiva es una causa de estrés estudiantil, incluso en los niveles más elevados (Montalvo y Montiel, 2020). No podemos pensar que una clase a distancia es igual que una presencial. De hecho, cuando damos clase presencial siempre surgen interrupciones, dudas, conflictos de grupo, etcétera, que no impiden impartir todos los conocimientos que teníamos originariamente previstos. No podemos usar la clase a distancia para adelantar todo el temario que no pudimos dar en el aula.

\section{Tener en consideración la situación personal y anímica del alumnado}

En estos momentos estamos viviendo un luto colectivo, que deriva del contacto estrecho con la muerte en muchas familias y que afecta el proceso de aprendizaje. Por un lado, la enfermedad que pudieran padecer los alumnos o sus familiares entorpece y puede cortar el proceso de aprendizaje de manera temporal. La flexibilidad, en este sentido, es esencial para un buen resultado. Por otro lado, el confinamiento domiciliario genera problemas de estrés postraumático y ansiedad, además falta de movilidad, que debe ser paliada, y se ha de controlar la alimentación (Andreu, 2020).

Otro de los grandes problemas es la sobreinformación de los medios, a la que el alumnado está expuesto sin ser capaz de entender las noticias o cribar las

${ }^{1}$ Noticia falsa que se disemina con un fin en específico. verídicas de los meros bulos ${ }^{1}$ (Paricio y Pando, 2020). Estos factores inciden en la disposición para el aprendizaje significativo, y es necesario mitigarlos con una 
acción tutorial mucho más estrecha hacia el alumnado, con el fin de detectar los casos de ansiedad y aislamiento. Por último, no debemos olvidar que el confinamiento agudiza las diferencias sociales, especialmente en el caso de los alumnos más vulnerables que tienen que quedarse solos en casa o habitar viviendas sin espacio suficiente.

En definitiva, debemos cambiar el punto de mira, reformular todo lo que habíamos planeado y ser conscientes de que los perfiles de alumno y profesor han cambiado por completo en estos días. De esta manera, haremos un trabajo mucho más efectivo.

\section{Conclusiones}

Los primeros datos estadísticos arrojan insatisfacción por parte del alumnado de diversos ámbitos (Lozano et al., 2020) debido a la situación de indefensión ante el cambio brusco. El problema es real y parece que no va a tener una pronta solución. En estos momentos la educación en todos los ámbitos ha sido afectada por la pandemia y debemos seguir enseñando de la mejor manera posible. Las pautas presentadas en este artículo, si bien están basadas en mi experiencia en secundaria, pueden ser adaptadas a todos los ámbitos educativos. Contemplan una fase previa de diagnóstico del perfil del profesor y del alumno, de sus fortalezas y carencias, en función de su entendimiento y manejo de los recursos digitales. Para una relación adecuada con el alumno, es esencial conocer los hábitos previos y la relación con las redes sociales, con el objetivo de ofrecer tareas adaptadas a los tiempos de atención y a los recursos en línea, de acuerdo con los diferentes dispositivos disponibles. Finalmente, ha de ser valorado el impacto psicológico del momento en el que vivimos, en especial entre los alumnos, y tenerlo en consideración en el desarrollo y evaluación de contenidos.

\section{Referencias}

* Andreu Cabrera, E. (2020). Actividad Físicay Efectos Psicológicos Del Confinamiento Por Covid-19. International Journal of Developmental and Educational Psychology Revista INFAD de Psicología, 2(1), 209-20, http://www.infad.eu/RevistalNFAD/OJS/ index.php/IJODAEP/article/view/1828/1612

* Duran, J. y Uribe Sandoval, A. (2011). El uso de las tics y la brecha digital entre adultos y adolescentes. Primer avance de resultados. https://www.researchgate. net/publication/273347869_EI_uso_de_las_TICs_y_la_brecha_digital_entre_ adultos_y_adolescentes_Primer_avance_de_resultados

* Cabero Almenara, J. (2014). Reflexiones sobre la brecha digital y la educación. http://diversidad.murciaeduca.es/tecnoneet/docs/2004/jcabero04.pdf

* Crovi Druetta, D. M. (2010, mayo-agosto). Jóvenes, migraciones digitales y brecha tecnológica. Revista Mexicana de Ciencias Políticas y Sociales, 52(209), 119-133. https://www.redalyc.org/articulo.oa?id=42116235008 
* Duart, J. M. (2010). Nuevas brechas digitales en la educación superior. Rusc. Universities and Knowledge Society Journal, 7(1), pp. 1-2. https://www.redalyc.org/ articulo.oa?id=78012953001

Johnson, S. (2005). Everything bad is good for your, how popular culture is actually making us smarter. Riverhead.

Mead, M. (1935). Sex and Temperament in Three Primitive Societies. William Morrow and Company.

* Montalvo Romero, N. y Montiel Rosales, A. (2020). Impacto del covid-19 en el estrés de los universitarios. Ava Cient, 4(2). http://itchetumal.edu.mx/avacient/ index.php/revista/article/view/132

* Lozano Díaz, A., Fernández-Prados, J. S., Figueredo Canosa, V. y Martínez Martínez, A. M. (2020). Impactos del confinamiento por el covid-19 entre universitarios: Satisfacción Vital, Resiliencia y Capital Social Online. International Journal of Sociology of Education, Special Issue: CoviD-19 Crisis and Socioeducative Inequalities and Strategies to Overcome them, 79-104. Dol: http://doi.org/10.17583/ rise.2020.5925

* Paricio del Castillo, R. y Pando Velasco, M. F. (2020, abril-junio). Salud mental infanto-juvenil y pandemia de Covid-19 en España: cuestiones y retos. Revista de Psiquiatría Infanto-Juvenil, 37(2), 30-44. Dol: https://doi.org/10.31766/revpsij. v37n2a4

* Piscitelli, A. (2006, enero-marzo). Nativos e inmigrantes digitales ¿Brecha generacional, brecha cognitiva, o las dos juntas y máss aún? Revista Mexicana de Investigación Educativa, 11(28), 179-185. https://www.redalyc.org/articulo. oa?id=14002809

- Piscitelli, A. (2009). Nativos digitales. Dieta cognitiva, inteligencia colectiva y arquitecturas de la participación. Santillana.

* Prensky, M. (2001, octubre). Digital Natives, Digital Immigrants. On the Horizon, 9(5). $\quad$ http://www.marcprensky.com/writing/Prensky\%20-\%20Digital\%20 Natives,\%20Digital\%20Immigrants\%20-\%20Part1.pdf [versión en español en http://docs.google.com/View?docid=ddttrkpp_29c595pr]

* Lamschtein, S. (2010). Las tics y la brecha generacional. http://dspace.mides.gub. uy:8080/xmlui/handle/123456789/628

\section{Cómo CitAR ESTE ARTículo}

* Duce Pastor, Elena. (2021, enero-febrero). La educación ante una pandemia: claves iniciales para superar brechas y poner manos a la obra. Revista Digital Universitaria (RDU), 22(1). Dol: http://doi.org/10.22201/cuaieed.16076079e.2021.22.1.1 